\title{
THE GROWTH HORMONE TREATMENT RESULTS IN THE INCREASE OF IRISIN CONCENTRATION IN PLASMA
}

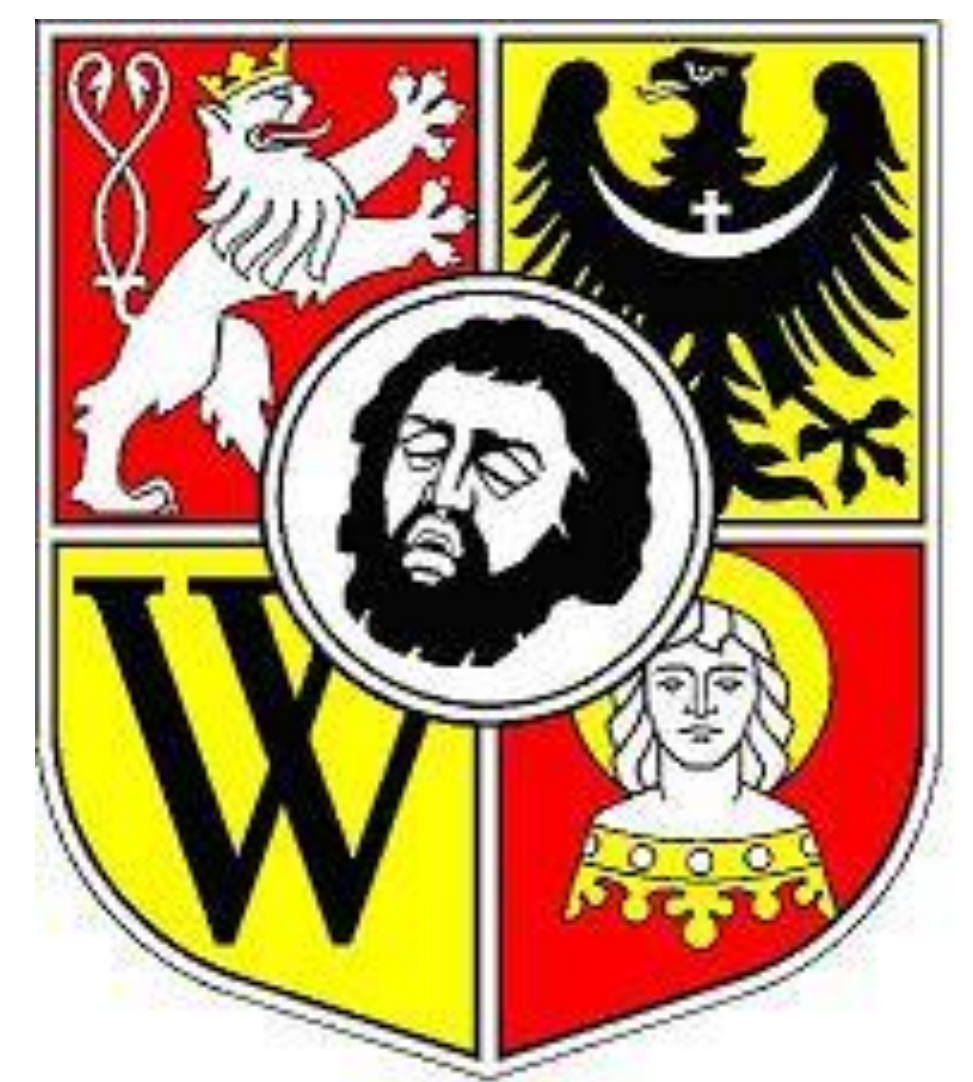

WMU

Wikiera Beata ${ }^{1}$, Pukajło Katarzyna ${ }^{2}$, Kaczmański Kukasz ${ }^{2}$, Natalia Słoka ${ }^{2}$, Aleksander Basiak ${ }^{1}$, Noczyńska Anna ${ }^{1}$, Bolanowski Marek ${ }^{2}$, Daroszewski Jacek ${ }^{2}$

1 Department of Endocrinology and Diabetes for Children and Adolescents, Wroclaw Medical University, Chałubińskiego 2, 50-368 Wroclaw, Poland

${ }^{2}$ Department of Endocrinology, Diabetes and Isotope Therapy, Wroclaw Medical University,

Pasteura 4, 50-367 Wroclaw, Poland

email: katarzyna.pukajlo@umed.wroc.pl

\section{Introduction}

Brown adipose tissue metabolism is of remarkable pathophysiological interest, because it could be a target for future therapies for obesity and metabolic syndrome.

Irisin (Ir), recently identified novel adipomyokine is essential in a white-tobrown fatty tissue transdifferentiation, and mediates some of the positive influences on metabolic disorders through increase of energy expenditure.

The exact regulation of $\mathrm{Ir}$ secretion and action is unknown but significant positive associations of circulating Ir with growth hormones and IGF-1 were found.

We studied Ir response in a group of Turner Syndrom (TS) patients treated with supraphysiological doses of growth hormone $(\mathrm{rGH})$.

\section{Study group and rGH treatment}

The study group consisted of 36 patients with Turner syndrome (TS) diagnosed in one pediatric tertiary center. Their median age was 7,06 years (range 3.2-16.07 years). The patients were diagnosed by karyotyping. Conventional G-banding and fluorescence in situ hybridization on peripheral blood cultures confirmed numerical or structural abnormalities of $X$ chromosome or mosaicism. $\mathrm{X}$ chromosome monosomy was established in 19 patients $(52,8 \%)$.

Because of short stature the patients were treated with $\mathrm{rGH}$ given subcutaneously once daily at bedtime, in a dose 0,05 $\mathrm{mg} / \mathrm{kg} /$ day. The dose of $\mathrm{rGH}$ was adjusted to body weight every 3 months.

The mean treatment period was $1.5 \mathrm{yr}$ (range 0.4-4.0yr). No other medication including estrogen replacement therapy was conducted during the study. Patients with coexisting endocrine diseases or other conditions interfering with glucose metabolism were excluded.

\section{Measurements}

Prior to and following the $\mathrm{rGH}$ treatment $(\mathrm{rGHt})$ anthropometrical data were recorded as well as biochemical parameters were measured: Ir, OGTT, insulin, IGF-1, and IGFBP-3.
The description of TS group and the effect of rGH therapy

\begin{tabular}{|ccc} 
& & \\
\hline & before rGHt & after rGHt \\
\hline Height [cm] & $112.14 \pm 16.3$ & $126.14 \pm 15.29$ \\
\hline Height SDS & $-2.45 \pm 0.91$ & $-1.8 \pm 0.83$ \\
BMI SDS & $0.18 \pm 1.27$ & $0.34 \pm 1.1$ \\
\hline $\begin{array}{c}\text { Fasting plasma } \\
\text { glucose } \\
\text { [mg/dl] }\end{array}$ & $87.77 \pm 9.13$ & $86.69 \pm 15.26$ \\
\hline $\begin{array}{c}\text { Fasting plasma } \\
\text { insulin }\end{array}$ & $3.67 \pm 2.3$ & \\
[ulU/ml] & & \\
\hline IGF-1 [ng/ml] & $119.40 \pm 62.47$ & $439.08 \pm 209.91$ \\
\hline IGF-1 SDS & $-1.70 \pm 0.48$ & $0.41 \pm 1.29$ \\
\hline IGFBP3 [ug/ml] & $3.94 \pm 0.83$ & $5.73 \pm 1.2$ \\
\hline & & \\
\hline
\end{tabular}

\section{Results}

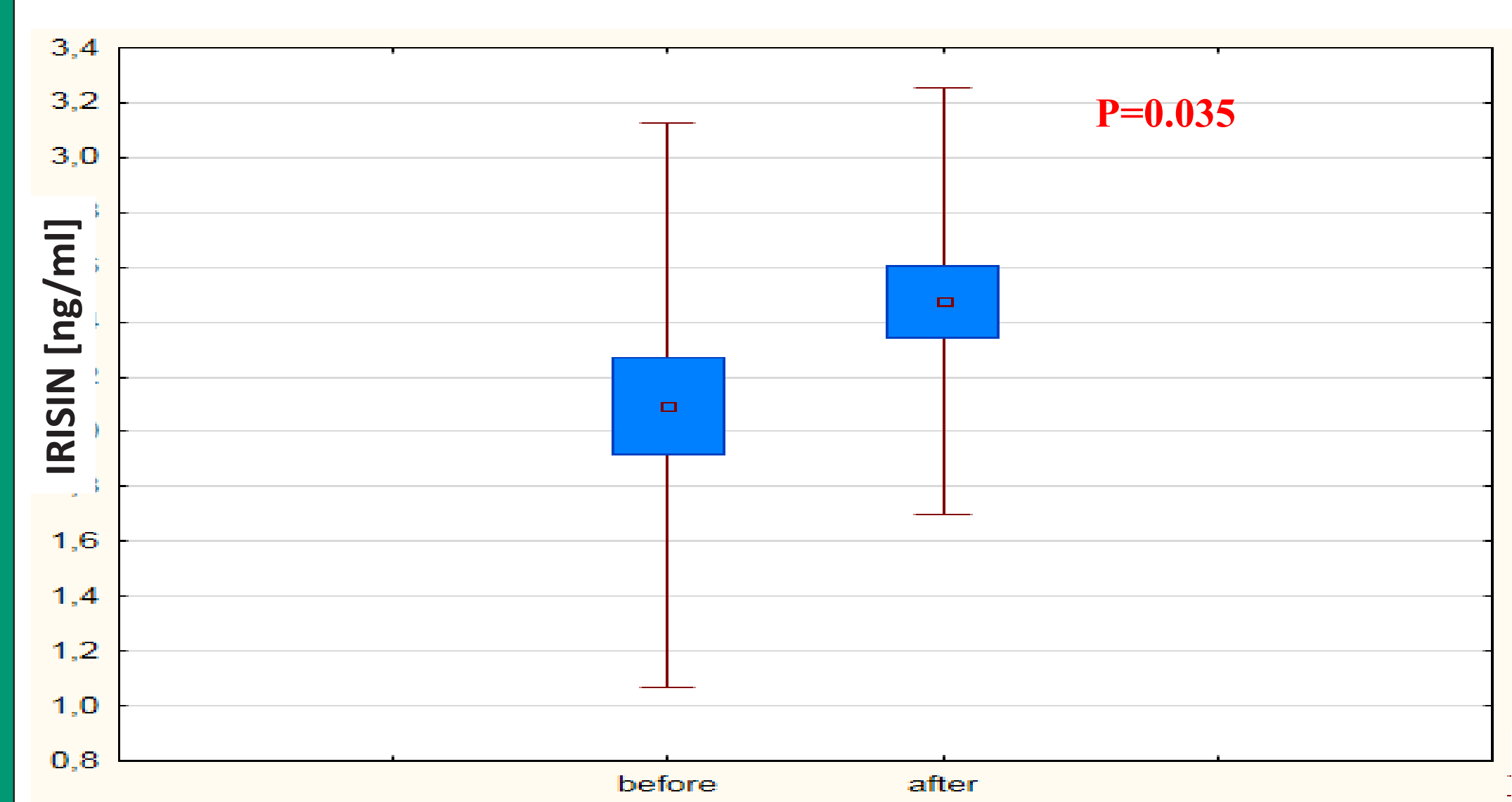

Irisin concentration increased significantly after rGHt

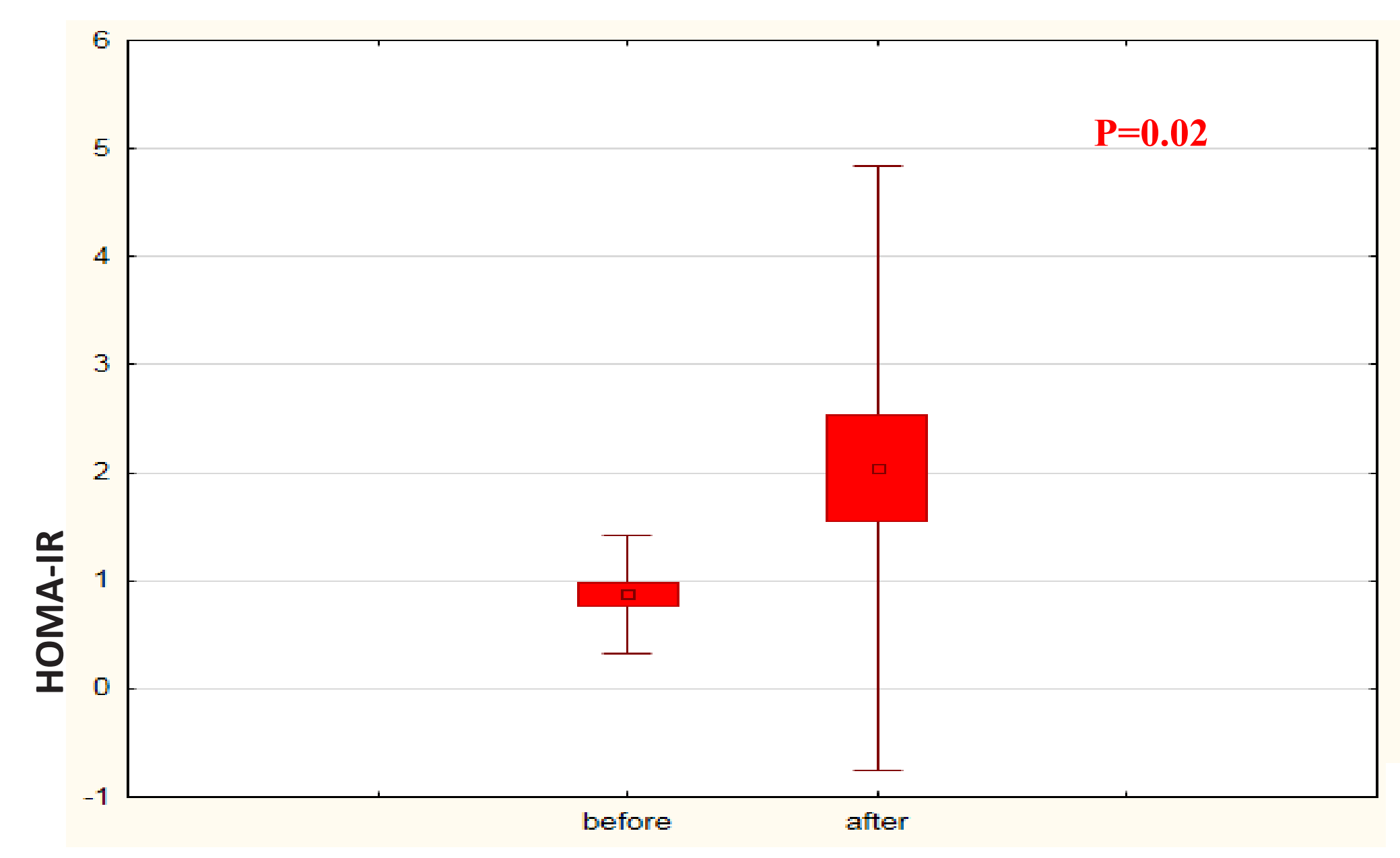

We observed the increase of HOMA-IR value at the end of $\mathrm{rGHt}$

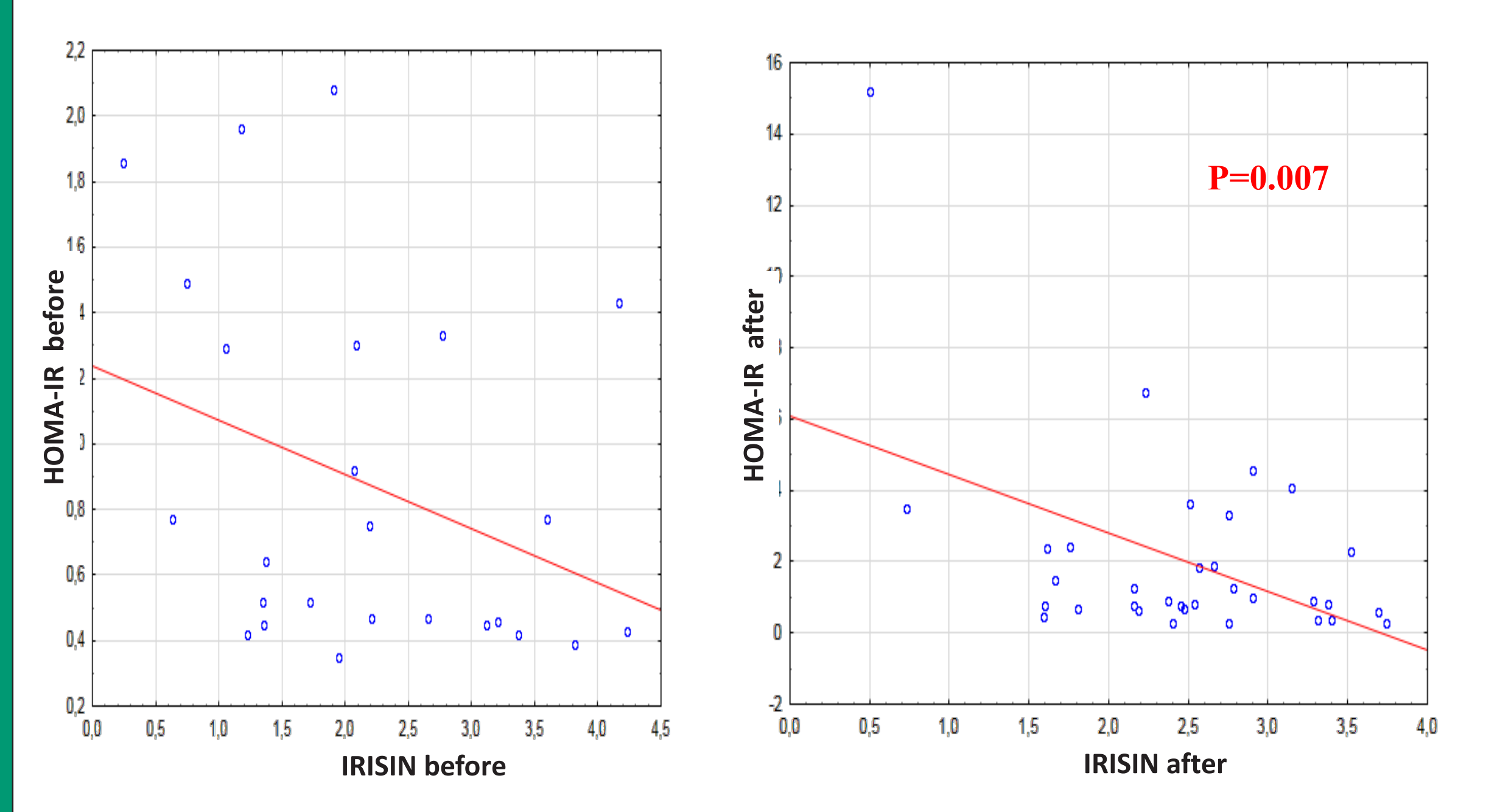

There was a negative association between Ir concentration and HOMA-IR after rGHt

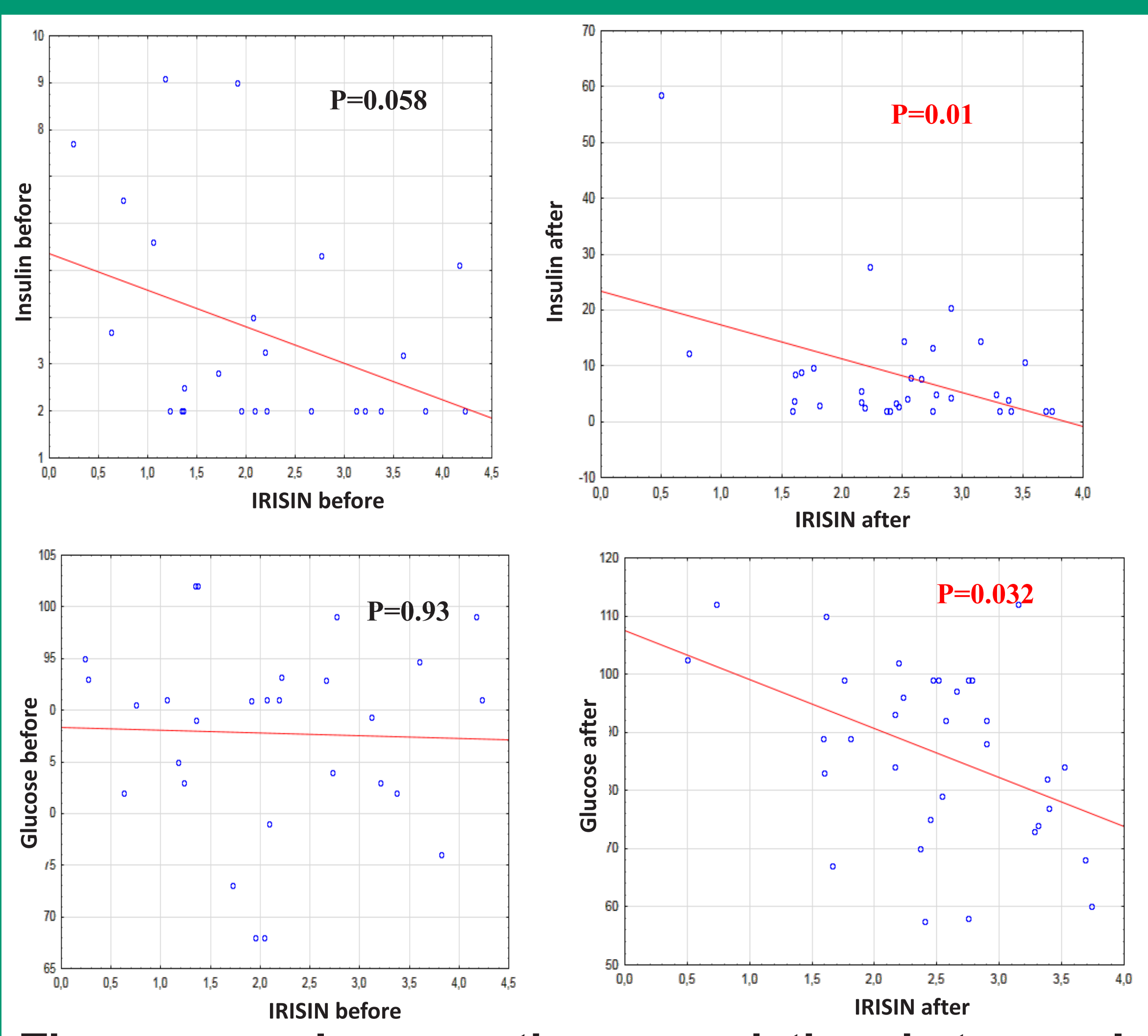

There was also negative association between Ir concentration and fasting insulin and glucose levels after $\mathbf{r G H t}$

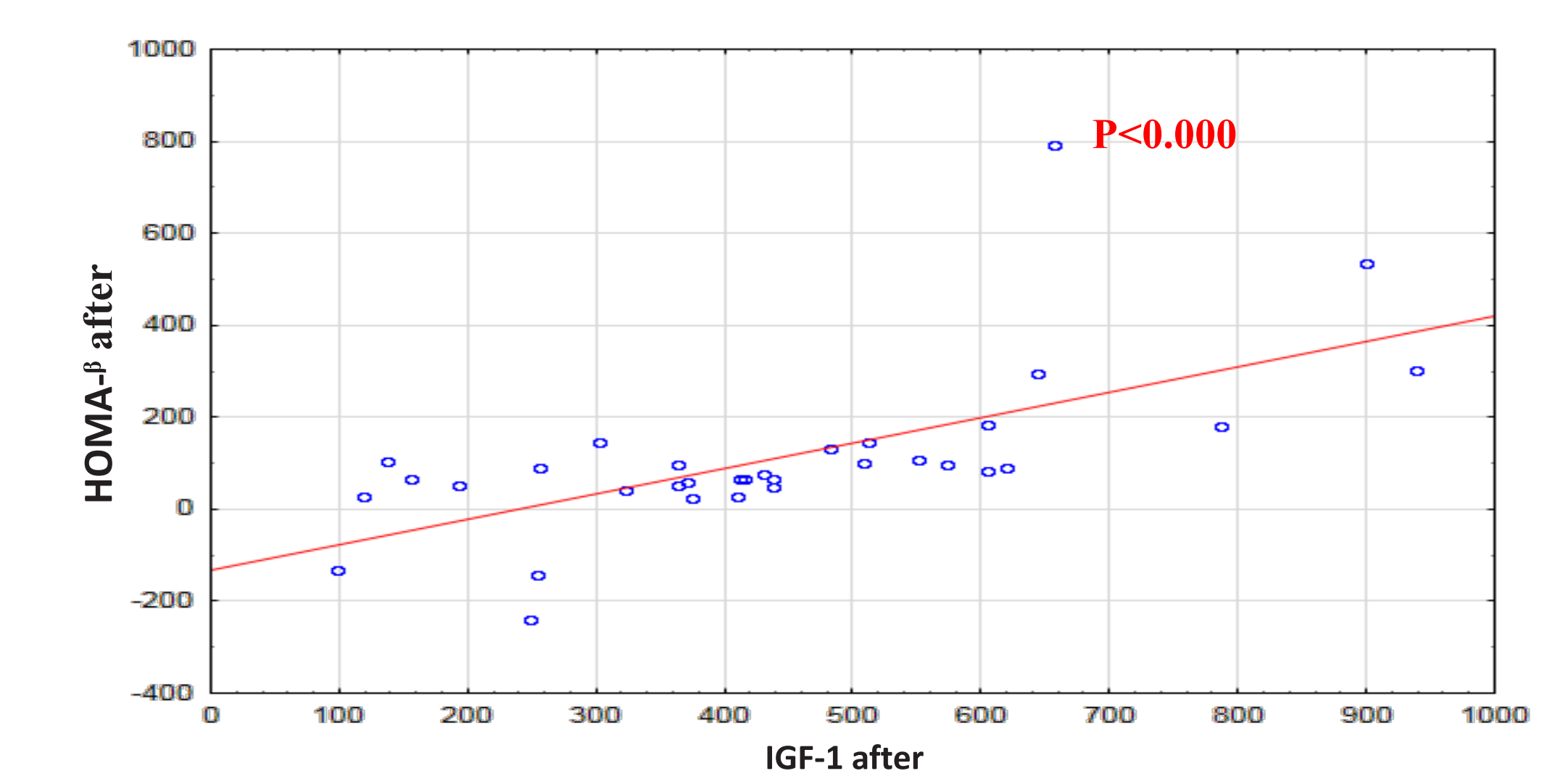

After $\mathrm{rGHt}$ a pronounced HOMA $\beta$ value increase related to IGF-1 level was observed but there was no association between Irisin concentration and HOMA- $\beta$ before or after rGHt.

\section{Conclusions}

1) rGH therapy has been shown to increase the concentration of circulating Irisin

2) $\mathrm{rGH}$ treatment seems to restore physiological function of Irisin

3) This study has shown that in the Turner Syndrome group the rGH medication improved $\beta$-cell function

4) No relation between Ir and IGF-1 levels or IGF-1/ IGFBP3 ratio was recorded, therefore the Irisin rise seemed not to be IGF-1 mediated.

5) The complex functional interrelationships between GH, IGF-1 and insulin resistance/sensitivity must be a subject of further investigations. Our data have shown that Irisin may be a new relevant player in this branch of energy metabolism.

To the best of our knowlege this is the first study on Irisin in Turner Syndrome patients. 